\title{
An Efficient Image Independent Thinning Algorithm
}

\author{
A. Jagna \\ Professor, Department of Computer Science \& Engineering, B.V.Raju Institute of Technology, Narsapur, Medak, \\ Telangana, India
}

\begin{abstract}
As we know that image thinning plays an important role in image processing, It is also equally important to propose an efficient image thinning algorithm with an objective, to minimize the amount of information to be processed by preserving the important information required to preserve the topological and geometrical properties of the thinned image, thereby enhancing the later processing procedure. This can be achieved by an efficient image independent thinning algorithm. This algorithm process the image in two-passes, in first pass of this algorithm, the entire image is thinned to two pixels thick and in second pass; the two pixel width image is further thinned to one pixel thick without any discontinuities in the resultant image.
\end{abstract}

Keywords: Iterative, parallel, topological, geometrical, enhancing.

\section{INTRODUCTION}

Thinning algorithms are developed to address wide variety of problems in thinning. These thinning algorithms are classified based on the approaches used for thinning [Lam, Lee \& Suen, 1992]. Based on these approaches thinning algorithms are classified as iterative and non-iterative thinning algorithms. With Non-iterative thinning algorithms skeleton can be obtained in a single pass (With the methodologies like distance transforms and run-length coding). But this algorithm, does not consider the surrounding pixels while making decisions for deletion or to retain. Whereas Iterative thinning algorithms considers the surrounding pixels while making decision for deletion or to retain pixels. The iterative thinning algorithms are further categorized as sequential (boundary pixels are processed one by one, layer by layer) and parallel (all the boundary pixels are processed simultaneously layer by layer). Sequential algorithms consume less amounts of memory. In contrast, parallel algorithms require more memory compared to sequential algorithms but are much faster in processing. Parallel algorithms process all the border pixels at once.

\section{LITERATURE REVIEW}

This paper covers the literature review on various parallel and sequential image thinning algorithms, such as order independent [1], [2], Rotation invariant [3], Rotation invariant rule based [4], and parallel [5]. In rotation invariant thinning, it is observed that, some lines containing only two pixel widths are completely deleted. In rotation invariant rule based thinning algorithms, the parallel application of the rules to each pixel in the image resulting in peeling off the outer and inner boundaries of symbols. However, some cases of two-pixels of wider linesare unfortunately deleted. An iterative parallel thinning algorithm [6], which uses four passes per iteration, thins down the pattern isotropically.The thinning algorithms [1], [5] are very popular because of their implementation simplicity and high computational speed.
But, the results they produce have drawbacks such as corners and endpoints are not considered for thinning. Further the distortions like spikes effect the subsequent operations. The results produced by [2], [3], and [4] are not a one pixel thick images and have discontinuities in the image pattern. This happens because of two-pixel width line is considered to be the outer boundary. Hence, its elimination causes discontinuities in the image. In rotation invariant rule based thinning algorithms, the parallel application of the rules to each pixel in the image resulting in peeling off the outer and inner boundaries of symbols. However, some cases of two-pixels or wider lines are unfortunately deleted. The drawback of the above procedure is that it results in discontinuous central lines. An iterative parallel thinning algorithm [6], which uses four passes per iteration, thins down the pattern isotropically.

Many thinning algorithms have been studied in [9], for their performance, and evaluation. None of the previous algorithms are image independent. Many are specific to digits, characters, or letters written in English, Chinese, or any other script. To overcome the above mentioned drawback, image independent thinning algorithm is proposed. This approach is generic and it can be used to thin symbols, digits, characters, or letters written in English, Chinese, Hindi and Telugu, irrespective of the script they are written in

\section{III.PROPOSED THINNING ALGORITHM}

The proposed approach is a two pass image independent thinning algorithm, which uses $3 \times 3$ masks to determine the thinning rules, these thinning rules are applied on each and every pixel for marking which pixels are to be deleted and which are to be retained in the resultant skeleton.

\section{A. Principle followed in the algorithm}

It is a two pass iterative parallel thinning algorithm. In this we adopt a two-pass thinning procedure which checks 
whether any black pixel can be removed (made to be white) safely or not. The pass-1 rules are nothing but an exhaustive list of alternative possible masks obtained by rotating the masks shown in figure 1.

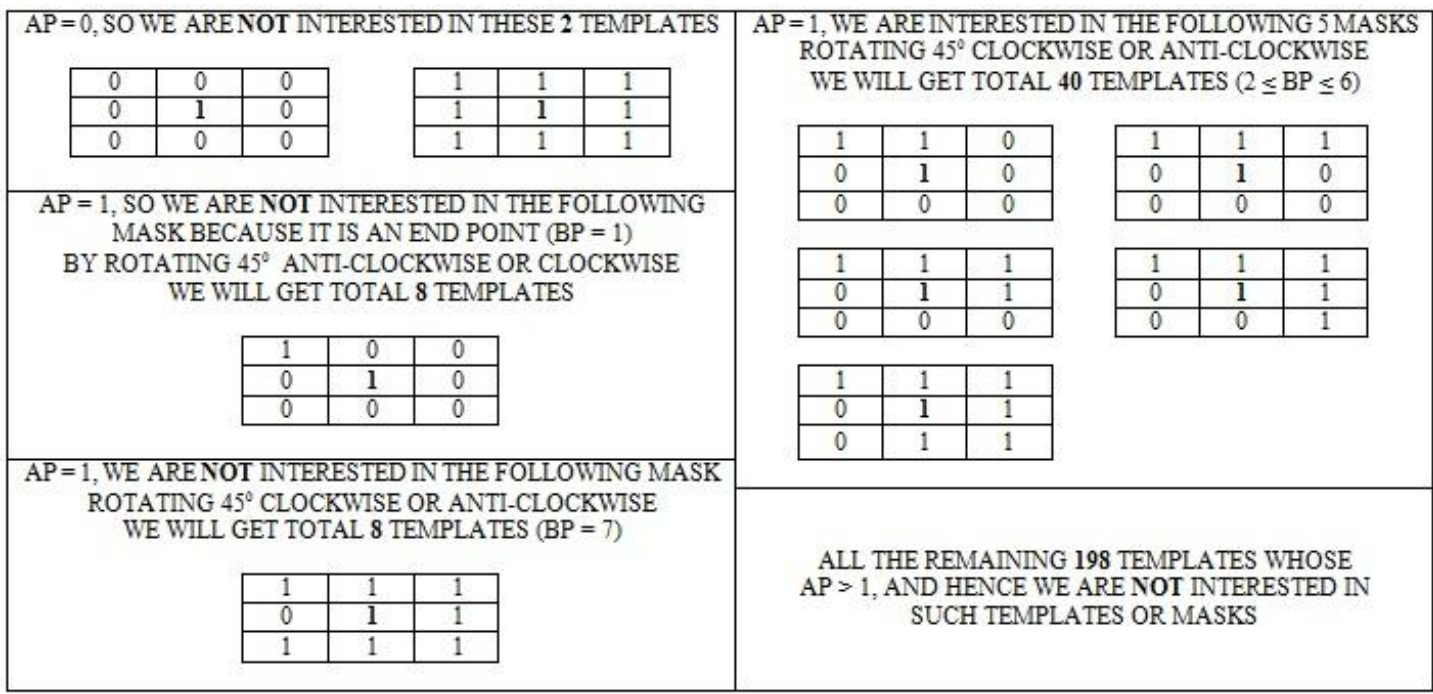

Figure 1.Masks used to derive the thinning rules for pass-

The masks shown in figure 1, are with $\mathrm{AP}=1$ and $\mathrm{BP}$ Pass-2:

value ranging from 3 to 6 respectively. By rotating each of these masks $45^{\circ}$ clockwise or anti-clockwise, we will get a total of 32 different masks. These masks are used to thin the image without breaking connectivity in the first pass. By enumerating all possible binary patterns of length 8 gives 256 distinct patterns. Accordingly there are 256 masks possible. Out of which we are interested in only 32 masks.

In pass-1, the proposed thinning algorithm simultaneously processes all the boundary pixels at once. If the pixel matches with deletion conditions of pass-1, it can be concluded that this pixel is on the boundary and should be deleted. This process is repeated until no more changes occur in pass-1. However, at the end of pass-1, the entire skeleton is of 2-pixel wide.

In pass-2, we initially Count the number of non-zero neighbors (black pixels-BP) of the candidate pixels $\mathrm{P}_{\mathrm{i}}$. If the count is three-to-five( 3 to 5 ), then the 2-pixel wide lines in the provisional skeleton produced by the pass-1 will be further thinned to one pixel wide skeleton, without compromising in the quality of thinned pattern.

B. Steps in the Algorithm

\section{Pass-1}

Step-1:

Count the number of non-zero neighbours and zero-to-one or one-to-zero transition of the (black pixel) candidate pixel Pi.

Step-2:

If the non-zero neighbours are in the range of two (2) to six (6) and either one-to-zero or zero-to-one transitions are exactly one (1), then process the pixel under consideration.

Step-3:

Iterate, step 1 and 2 until no more changes take place in

Step-4:

Count the number of non-zero neighbours (black pixels$\mathrm{BP})$ of the candidate pixels Pi. If the count is three-to-five, then process the pixel under consideration.

Step-5:

Repeat the step- 4 until no more change take place in pass- 2 .

\section{IV.RESULTS AND COMPARISONS}

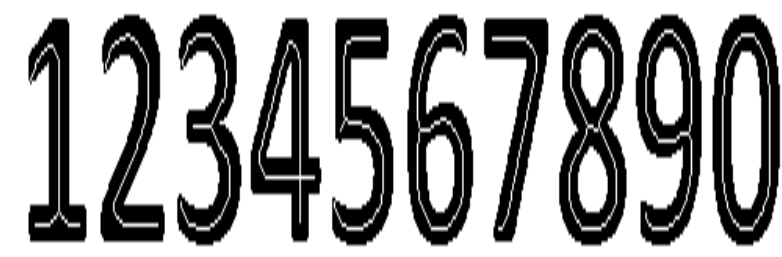

The results of the proposed algorithm are compared with ZS and KNP thinning algorithms in terms of excessive erosion, connectivity, thickness and symmetry of the skeletons, endpoint preservation, and visual quality. It is also found to be superior to the existing algorithms in all the above characteristics. The figure 2 to figure 6 is thinned with the proposed thinning algorithm and results are shown below.
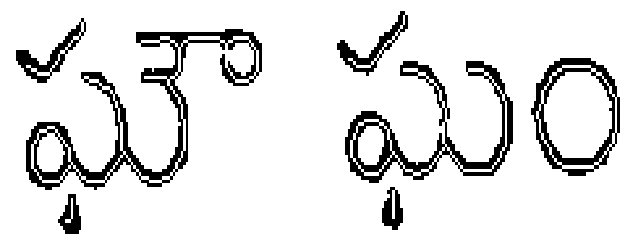

Figure 2. Telugu Characters thinned with proposed thinning Algorithm pass-1, else goto pass- 2 . 


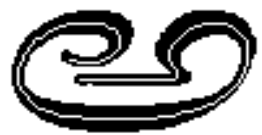

Figure 3. Telugu Character Aa thinned with proposed thinning Algorithm

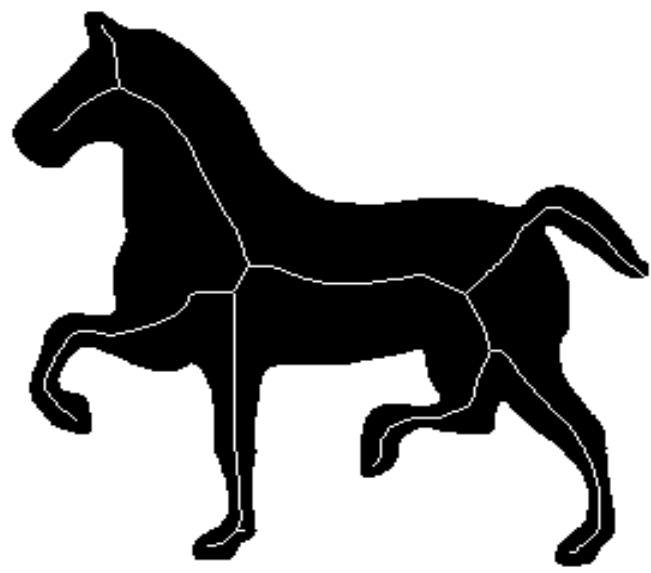

Figure 4. Horse image thinned with proposed thinning Algorithm

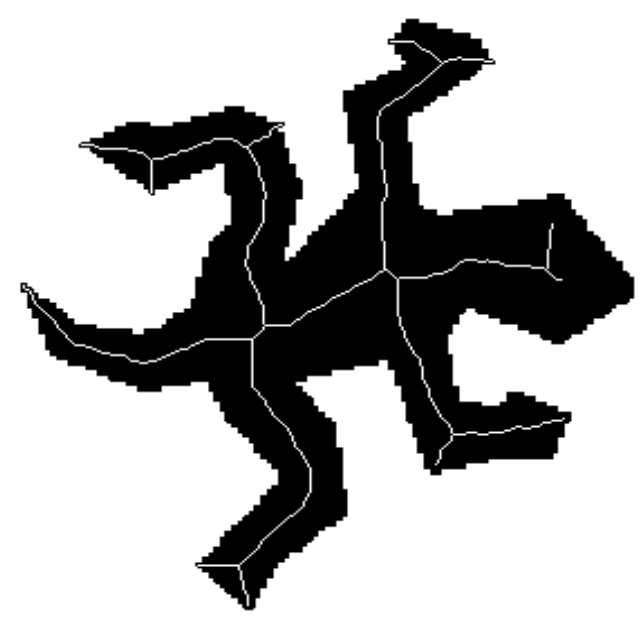

Figure 5. Lizard image thinned with proposed thinning Algorithm

Table 1: Comparison of thinning time and thinning rate

\begin{tabular}{|c|c|c|c|c|c|c|}
\hline \multirow{2}{*}{$\begin{array}{l}\text { Input } \\
\text { Image }\end{array}$} & \multicolumn{3}{|c|}{$\begin{array}{l}\text { Thinning Time } \\
\text { in milliseconds }\end{array}$} & \multicolumn{3}{|c|}{$\begin{array}{l}\text { Thinning Rate }(\%) \\
\text { (Intel C2D } 160 \mathrm{GHz} \\
\text { Processor with } 512 \mathrm{MB} \text { RAM) }\end{array}$} \\
\hline & $\begin{array}{l}\mathrm{ZS} \\
\text { Alg. }\end{array}$ & $\begin{array}{l}\text { KNP } \\
\text { Alg. }\end{array}$ & $\begin{array}{l}\text { Prop } \\
\text { Alg. }\end{array}$ & $\begin{array}{l}\mathrm{ZS} \\
\text { Alg. }\end{array}$ & $\begin{array}{l}\text { KNP } \\
\text { Alg. }\end{array}$ & $\begin{array}{l}\text { Prop } \\
\text { Alg. }\end{array}$ \\
\hline Fig.6(a) & 74.35 & 65.01 & 63.54 & 88.46 & 91.75 & 95.54 \\
\hline Fig.6(b) & 269.50 & 134.75 & 130.50 & 83.32 & 89.47 & 93.38 \\
\hline Fig.6(c) & 16.00 & 15.00 & 15.00 & 89.58 & 92.30 & 94.75 \\
\hline Fig.6(d) & 32.00 & 15.89 & 15.75 & 90.26 & 93.98 & 95.35 \\
\hline Fig.6(e) & 28.26 & 15.54 & 15.38 & 89.39 & 93.27 & 95.15 \\
\hline
\end{tabular}

Image thinning has been performed on a few sample images using order independent thinning algorithm (KNP) and parallel thinning algorithm (ZS). The results show that KNP algorithm is not able to thin the image comprehensively; which means that it is leaving some parts of the image. In most of the cases, the edges and the corner points are not processed. In ZS algorithm there is excessive erosion and discontinuities in the thinned pattern are observed, where as proposed algorithm has processed the entire image and produced the exact skeleton for the given input image. A unique advantage of the proposed algorithm is that this algorithm does not change the topological properties of the connectivity and the shape of the thinned image when compared to the other existing algorithm.

\section{CONCLUSION}

We have proposed an efficient image independent parallel thinning algorithm. Implementation of this algorithm has been carried out and we have also compared its results with other standard thinning algorithms in terms of thinning time, thinning ratio, excessive erosion, connectivity, endpoint preservation, and visual quality. Results indicate that the proposed algorithm is efficient. The robustness of the proposed thinning algorithm has been established across heterogeneous image examples.

\section{REFERENCES}

[1] Peter Kardos, Gabor Nemeth, and Kalman Palagyi "An order independent sequential thinning algorithm”, IWCIA 2009, LNCS 5852, pp. 162-175, 2009@ Springer - Verlag Berline Heidelberg 2009.

[2] [2] Marcine Iwanowski and Pierre Soille, “ Order Independence in Binary 2D Homotopic Thinning”, A.kuba, L.G. Nyul, and K Palagyi(Eds): DGCI 2006, LNCS 4245,pp. 592-604,2006, (C) Springer - Verlag Berline Heidelberg 2006.

[3] Pradeep M.Patil, Shekar R.Suralkar, Faiyaz B.Sheikh,’Rotation Invariant Thinning to Detect Ridges Bifurcations for Fingerprint Identification", ICTAI-05, IEEE computer Society ,10823409,2005.

[4] Maher Ahmed and Rabab Ward," A Rotation Invariant Rule-Based Thinning Algorithm for Character Recognition”, IEEE Transaction on Pattern Analysis and Machine Intelligence, Vol.24, pp.no.16721678, No.12, Dec-2002.

[5] Zhang T Y and Suen C Y (1984), "A Fast Parallel Algorithm fo Thinning Digital Patterns", Research Contributions, Communications of the ACM, Vol. 27, No. 3, pp. 236-239.

[6] C.Arcelli, P.C.K.Kwok, G Sanniti di Baja,"Medial Lines by Parallel Wise Erosion of Successive Contour Pairs", Proc. IEEE Int'l Conf. TENCON, Technology Enabling Tomorrow, vol.1, pp.66-70, 1992.

[7] Ranwz. V, Soille. P, "Order Independent homotopic thinning for binary and gray tone anchored skeletons". Pattern Recognization Letters 23,687-702(2002).

[8]Gulshan Goyal, Maitreyee Dutta and Er.Akshay Gridhar, "A Parallel Thinning Algorithm for Numerical Pattern Images in BMP Format", published in International Journal of Advanced Engineering \& Application, pp.no.197-202. Jan.2010.

[9]L.Lam and C.Suen, "An Evaluation of Parallel Thinning Algorithms for character recognition", IEEE Trans. Pattern Analysis and Machine Intelligence, vol.17, no.9, pp914-919, Sept.1995 